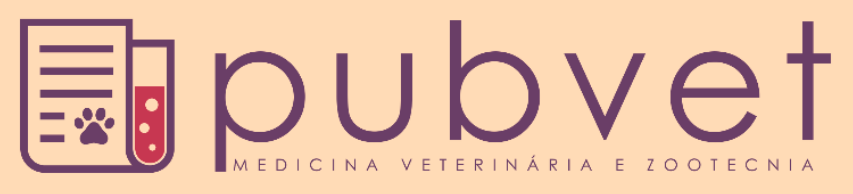

https://doi.org/10.31533/pubvet.v14n8a626.1-15

\title{
Situação da neosporose na bovinocultura brasileira
}

\author{
Charles Mendes de Oliveira ${ }^{\circ}$, Andresa dos Santos $\operatorname{Veras}^{10}$, Luis Fernando Faria Coury ${ }^{2}$, $^{\circ}$, \\ Lorena Aparecida de Bessa $^{2}{ }^{\circ}$, Renata Lima de Miranda ${ }^{3} \bullet$, Mariana Assunção de Souza $^{4}{ }^{\circ}$, \\ Jacqueline Ribeiro de Castro ${ }^{5 * 0}$
}

${ }^{1}$ Graduandos em Medicina Veterinária, Faculdade Presidente Antônio Carlos-UNIPAC-MG, Brasil.

${ }^{2}$ Graduados em Medicina Veterinária, Centro Universitário de Patos de Minas -UNIPAM-MG, Brasil.

${ }^{3}$ Médica Veterinária, Dra. em Parasitologia no Programa de Pós graduação em Imunologia e Parasitologia Aplicada -PIPPA- Universidade Federal de Uberlândia - UFU - MG, Brasil.

${ }^{4}$ Docente em Medicina Veterinária, Centro Universitário de Patos de Minas - UNIPAM - MG, Brasil.

${ }^{5}$ Docente em Medicina Veterinária, Centro Universitário do Triângulo - UNITRI-MG, Brasil.

*Autor para correspondência, E-mail: jack_ufu@yahoo.com.br

Resumo. A neosporose tem emergido no Brasil como uma das principais doenças causadoras de aborto em bovinos no Brasil e no mundo. Causada pelo protozoário Neospora caninum afeta uma diversidade de espécies animais. Em busca da elucidação biológica, epidemiológica e diagnóstica, vários estudos se delineiam a cerca desse agente. O objetivo do presente estudo foi revisar a literatura científica sobre a neosporose bovina no Brasil, abordando os principais aspectos etiopatogênicos, epidemiológicos, clínicos, diagnósticos e imunoprofiláticos, visando à construção do atual cenário da neosporose na bovinocultura brasileira. A estratégia de busca para seleção dos estudos foi um levantamento realizado nas bases de dados PubMed, Lilacs, SciElo e Google scholar, no período janeiro de 2005 a março de 2020. Parte majoritária dos estudos foram levantamentos soroepidemiológicas que visavam determinar a ocorrência e fatores de risco da doença. A ocorrência média da neosporose encontrada nos estudos foi de 32,7\% (2,45\%$100 \%$ ) associadas aos fatores de risco histórico de aborto, presença de cães, tipo de criação, idade e ausência de teste diagnóstico na introdução de novos animais nas propriedades. Os principias métodos diagnósticos utilizados foram os sorológicos. $\mathrm{O}$ aborto foi apontado como principal manifestação clínica da doença. A neosporose bovina está amplamente distribuída pelo Brasil, em alguns estudos de bovinocultura de exploração leiteira foram identificadas soroprevalências acima de $90 \%$ justificando-se assim, a implementação de estratégias de controle e profilaxia da neosporose bovina no Brasil, em busca de melhorias dos índices reprodutivos do rebanho nacional.

Palavra-chave: bovinos, Neospora caninum, protozoários, reprodução

\section{Situation of neosporosis in Brazilian cattle}

Abstract. Neosporosis has emerged in Brazil as one of the main diseases that cause abortion in cattle in Brazil and worldwide. Caused by the protozoan Neospora caninum affects a diversity of animal species. In search of biological, epidemiological and diagnostic elucidation, several studies are outlined about this agent. The goal of this study was to review the scientific literature on bovine neosporosis in Brazil, addressing the main etiopathogenic, epidemiological, clinical, diagnostic and immunoprophylactic aspects, aiming at building the current scenario of neosporosis in Brazilian cattle farming. The search strategy for selecting the studies was a survey carried out in the databases PubMed, Lilacs, SciElo and Google scholar, from January 2005 to March 2020. The majority of the studies were seroepidemiological surveys that aimed to determine the occurrence and factors of disease risk. The average occurrence of neosporosis found in the studies was 
$32.7 \%(2.45 \%-100 \%)$ associated with historical risk factors for abortion, presence of dogs, type of breeding, age and absence of diagnostic test when introducing new ones. animals on the properties. The main diagnostic methods used were serological. Abortion was identified as the main clinical manifestation of the disease. Bovine neosporosis is widely distributed in Brazil. In some dairy farming studies, seroprevalences above $90 \%$ have been identified, thus justifying the implementation of strategies for the control and prophylaxis of bovine neosporosis in Brazil, in search of improvements in reproductive rates of the national herd.

Keywords: bovines, Neospora caninum, protozoan, reproduction

\section{Situación de la neosporosis en la ganadería brasileña}

Resumen. La neosporosis ha surgido en Brasil como una de las principales enfermedades que causan el aborto en el ganado en Brasil y en todo el mundo. Causada por el protozoo Neospora caninum afecta a una diversidad de especies animales. En busca de aclaraciones biológicas, epidemiológicas y de diagnóstico, se describen varios estudios sobre este agente. El objetivo de este estudio fue revisar la literatura científica sobre la neosporosis bovina en Brasil, abordando los principales aspectos etiopatogénicos, epidemiológicos, clínicos, diagnósticos e inmunoprofilácticos, con el objetivo de construir el escenario actual de la neosporosis en la ganadería brasileña. La estrategia de búsqueda para seleccionar los estudios fue una encuesta realizada en las bases de datos PubMed, Lilacs, SiElo y Google Scholar, desde enero de 2005 hasta marzo de 2020. La mayoría de los estudios fueron encuestas serológicas epidemiológicas que tenían como objetivo determinar la casuística y los factores de riesgo de enfermedad La aparición promedio de neosporosis encontrada en los estudios fue del $32.7 \%(2.45 \%-100 \%)$ asociada con factores de riesgo históricos de aborto, presencia de perros, tipo de cría, edad y ausencia de pruebas de diagnóstico al introducir nuevos animales en las propiedades. Los principales métodos de diagnóstico utilizados fueron serológicos. El aborto se identificó como la principal manifestación clínica de la enfermedad. La neosporosis bovina está ampliamente distribuida en Brasil, en algunos estudios de producción lechera se han identificado seroprevalencias superiores al $90 \%$, lo que justifica la implementación de estrategias para el control y la profilaxis de la neosporosis bovina en Brasil, en busca de mejoras en las tasas reproductivas. del rebaño nacional.

Palabras clave: bovinos, Neospora caninum, protozoos, reproducción

\section{Introdução}

A sanidade dos rebanhos é um dos desafios constantes enfrentados pelo Brasil, o qual segue com o maior rebanho comercial do mundo, composto aproximadamente por 213,5 milhões de animais (ANUALPEC, 2019). O manejo sanitário é essencial no controle e profilaxia das doenças que afetam os índices produtivos e reprodutivos na bovinocultura. Nesse contexto, destacam-se as doenças de impacto reprodutivo, como a neosporose, a qual se configura no painel atual como uma das mais relevantes enfermidades causadoras de aborto na espécie bovina (Bruhn et al., 2012; Maldonado Rivera et al., 2020; Snak \& Osaki, 2019).

A neosporose é uma doença parasitária, causada pelo agente coccídeo Neospora caninum( $N$. caninum), um protozoário intracelular obrigatório, que foi identificado pela primeira vez na Noruega em 1984 (Dubey et al., 1988) em cães com manifestações neurológicas, os quais apresenta vamcistos em sistema nervoso central morfologicamente distintos de Toxoplasma gondii.Um protozoário heteroxeno, com necessidade de dois hospedeiros para completar seu ciclo biológico (Megid et al., 2016), pode infectar diversas espécies como bovinos, bubalinos, equinos, suínos, ovinos, cervídeos epicitacídeos (hospedeiros intermediários), sendo os canídeos, domésticos e silvestres, os hospedeiros definitivos detentores da reprodução sexuada do patógeno (Basso et al., 2010; $\underline{\text { Bruhn et al., 2012; }}$ Megid et al., 2016). 
A transmissão desse agente se dá basicamente por duas maneiras, a transmissão vertical e horizontal. Essa trata-se de uma transmissão em que os hospedeiros intermediários são infectados por ingerem os oocistos esporulados do hospedeiro definitivo no ambiente, enquanto que, àquela pode ocorrer pela transmissão endógena (recrudescimento da infecção, com reativação de bradizoítos maternos durante o período gestacional)ou exógena (via hematógenica-uterina de mães recém infectadas). Vale destacar a importância da transmissão transplacentária endógena na manutenção da doença no rebanho, uma vez que, cerca de $95 \%$ das vacas soropositivas irão abortar ou gerar bezerros soropositivos (Diniz et al., 2019; McAllister, 2016).

A principal manifestação clínica dessa doença em bovinos é o aborto que ocorre geralmente entre o quinto e o sexto mês de gestação (Megid et al., 2016; Varandas et al., 2001). Vacas infectadas podem apresentar em repetição de cio, com intervalos regulares ou irregulares, gestação a termo com bezerros natimortos, nascimento de animais fracos e inviáveis, e nascimento de bezerros com manifestações clínicas neurológicas apresentando encefalite, paralisias, ataxias motoras, má formações, pneumonite e miocardite (Almeria et al., 2010; Cerqueira-Cézar et al., 2017; Flores, 2012; Maldonado Rivera et al., $\underline{2020})$.

Os métodos utilizados para diagnosticar a neosporose em bovinos são sorológicos(Ensaio de Imunoadsorção Enzimático-ELISA, Reação de Imunofluorescência Indireta - RIFI,Teste de Aglutinação do Neospora- NAT, Teste de Imunocromatografia Rápida - RIT, Teste de Aglutinação em Latex - LAT e Immunoblotting), técnicas moleculares (Reação em Cadeia da Polimerase - PCR), histopatológicas (Imunoistoquímica- IHQ) e ainda por isolamento, podendo utilizar cultivo celular em camundongos (Carvalho et al., 2014; Margarido et al., 2008; Silva et al., 2013; Snak \& Osaki, 2019). O diagnóstico sorológico se sobrepuja no cenário atual por ser uma metodologia comumente utilizada em levantamentos soroepidemiológicos, como ferramenta de triagem da doença na propriedade rural.

Devido à neosporose ser ocorrente na bovinocultura brasileira, em algumas propriedades apresentar elevada ocorrência, acima de 85\% (Zanatto et al., 2019) e cursar com manifestações clínicas inespecífica com impactos significativos (Bruhn et al., 2012; Snak \& Osaki, 2019), tornam-se fundamentais e desafiadores, estudos que compilem e discutam de maneira crítica o cenário atual epidemiológico e aspectos a cerca dessa doença.

Dessa forma, objetivou-se realizar uma revisão de literatura sobre a neosporose bovina no Brasil, abordando os principais aspectos etiopatogênicos, epidemiológicos, clínicos, diagnósticos e imunoprofiláticos, visando à construção do atual cenário da neosporose na bovinocultura brasileira.

\section{Estratégia da busca}

A estratégia de busca para seleção dos estudos foi o levantamento realizado nas bases de dados PubMed, Lilacs, SciElo e Google scholar, com o uso dos descritores (cattle) AND (neospora) AND (Brazil), nas línguas inglesa e portuguesa, no período de janeiro de 2005 a março de 2020. No Pubmed foram levantados 141 artigos, Lilacs 43 artigos relacionados, 39 estudos no SciElo e no Google scholar 4.170 artigos.

Destas, foram selecionadas citações potencialmente relevantes e relacionadas com o tema da pesquisa pelo título e excluídas todas que abordavam outras espécies que não a bovina. Em seguida, foram avaliados os resumos e excluídos estudos repetidos no banco de dados e aquele que não atendiam o escopo da presente revisão. Após foram avaliados em sua totalidade apenas os artigos que contribuíram de forma objetiva na construção do cenário da neosporose no Brasil, bem como, abordassem pontos relevantes sobre aspectos etiopatogênicos, epidemiológicos, clínicos, diagnósticos e/ou imunoprofiláticos da doença. Foram também utilizadas fontes clássicas sobre o assunto.

\section{Etiopatogênese}

Sua classificação taxonômica é descrita como um protozoário pertencente do filo Apicomplexa, classe Sporozoasida, subclasse Coccidiasina, ordem Eucoccidionarida, família Sarcocystidae, subfamília Toxoplasmatinae, gênero Neospora, espécie Neospora caninum (Megid et al., 2016). 
Os três estágios morfológicos do $N$. caninum são taquizoítos, bradizoítos e esporozoítos, os quais se diferenciam conforme a fase do ciclo biológico. Os taquizoítos se caracterizam pela fase de multiplicação rápida, medem cerca de $7,5 \times 2 \mu \mathrm{m}$ e apresentam formato ovóide ou semilunar. Neste estágio, o coccídeo se divide assexuadamente por endodiogenia, e é eficiente em infectar uma ampla variedade celular dos hospedeiros, como hepatócitos, células renais, miócitos, neurônios, macrófagos, células endoteliais e fibroblastos (Megid et al., 2016).

Já os bradizoítos encontram-se no interior dos cistos teciduais, caracteriza-se pela fase de multiplicação lenta, encistados principalmente em tecido neural e muscular(Carvalho et al., 2014). Medem aproximadamente 6-8 x 1-1,8 $\mu \mathrm{m}$ envolto por um cisto circular de até $107 \mu \mathrm{m}$ de parede lisa com $4 \mu \mathrm{m}$ de espessura (Megid et al., 2016; Snak \& Osaki, 2019).

Tem como seu hospedeiro definitivo os canídeos que são capazes de realizar a fase de sexuada de gametogonia e de eliminarem oocistos não esporulados pelas fezes, que medem cerca de 11,7 x 11,3 $\mu \mathrm{m}$, que podem ser ingeridos pelo hospedeiro intermediário, representados pelos bovinos, ovinos, equinos, cervos, caprinos, ratos silvestres e raposas que infectam-se ingerindo oocistos esporulados no meio ambiente (Donahoe et al., 2015; Llano, 2013; Megid et al., 2016).

A patogenia do agente está diretamente relacionada com a interação parasita hospedeiro. Dependerá da virulência da espécie genética envolvida, relacionada com o poder de invasividade, da expressão das proteínas de roptrias e mecanismo de evasão do parasita. Em relação ao hospedeiro, características como, estado imunológico, espécie acometida, idade, fase gestacional, presença de comorbidades, sinalização de citocinas e respostas imune celular e de anticorpos modularão e determinarão a resposta frente à infecção. Nessa interação aspectos ambientais também serão relevantes, pois fatores como temperatura, tempo de exposição ambiental e umidade do ambiente determinarão a viabilidade de esporulação do oocisto que será a forma infetante desse agente (Cerqueira-Cézar et al., 2017; Megid et al., 2016; Snak \& Osaki, 2019).

\section{Ciclo biológico e transmissão}

Como mencionado anteriormente, o N. caninum possui em seu ciclo biológico três formas infectantes conhecidas, os taquizoíto, os esporozoítos presentes no interior dos oocistos e os bradizoítos, localizados no interior de cistos teciduais (Dubey et al., 2002).

O ciclo se inicia com a eliminação de oocistos não esporulados nas fezes do hospedeiro definitivo, representado pelos canídeos domésticos e silvestres (Figura 1), depois de eliminados no ambiente necessitam de cerca de 24 a 48 horas para se tornarem esporulados e consequentemente infectantes, pelo processo de esporogonia (Dubey et al., 2007). O oocisto esporulado é composto de dois esporocistos, com quatro esporozoítos cada (Megid et al., 2016).

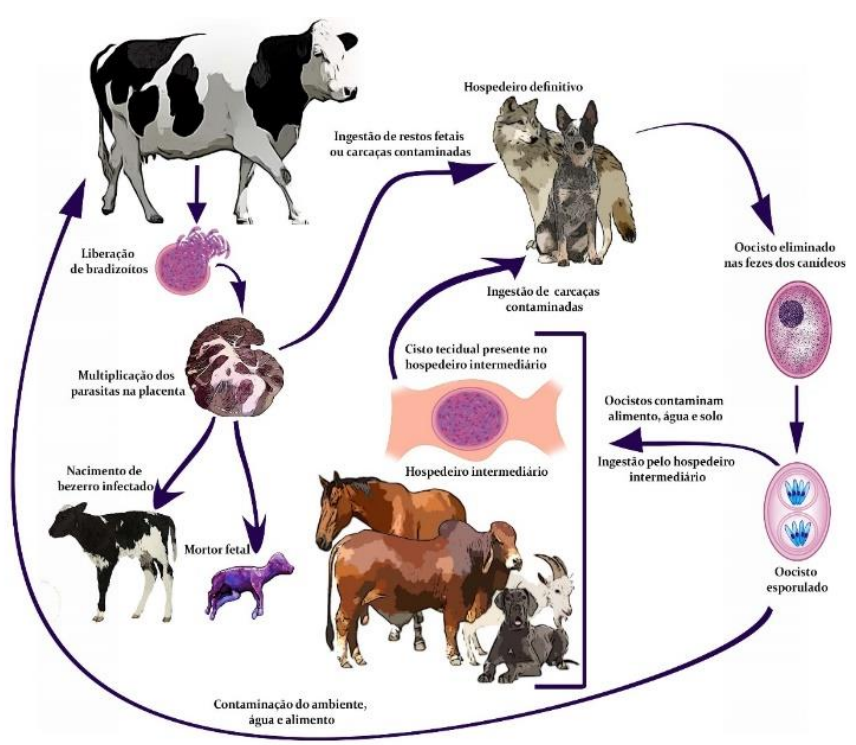

Figura 1. Ciclo biológico do Neospora caninum. 
O hospedeiro intermediário ingere o oocisto presente na pastagem ou água contaminada, forma de transmissão horizontal, então no trato digestório ocorre a liberação dos esporozoítos e penetração nas células intestinais, leucócitos e fibroblastos, para iniciar sua multiplicação, tornando-se taquizoíto, este por sua vez se multiplica e invade novas células podendo ser encontrados em células musculares, endotélio vascular, hepatócitos, células neurais e macrófagos, sendo todo esse processo de replicação ocorre de maneira assexuada (Dubey et al., 2002; Goodswen et al., 2013).

Após intensa multiplicação, os taquizoítos penetram principalmente em células do sistema nervoso central e musculatura, iniciando uma fase de proliferação mais lenta, onde o protozoário se encista na forma debradizoíto, esses parasitas ficam envoltos por um cisto de forma circular e parede lisa, uma forma de evasão e proteção do sistema imune, podendo permanecer no hospedeiro por toda sua vida, sem causar manifestações clínicas (Andreotti, 2001; Llano, 2013). O ciclo se completa após o hospedeiro definitivo ingerir o tecido com a presença de cistos do protozoário, liberando os bradizoítos no intestino, que invadem as células passando a se replicar de forma sexuada, por volta de cinco dias após a ingestão ocorre a liberação de oocistos não esporulados nas fezes (Almería \& López-Gatius, 2013). Outra forma de transmissão é a vertical, também denominada transplacentária que ocorre infecção da mãe para o feto, sendo a principal forma de transmissão na espécie bovina. Seu mecanismo ainda não é totalmente conhecido, no entanto, acredita-se que os taquizoítos passam da mãe para o feto através da placenta, podendo levar além da infecção fetal o aborto (Goodswen et al., 2013). Classificase em transmissão vertical endógena e exógena, nessa o hospedeiro é infectado durante a gestação e taquizoítos por via hematogéna vão até o feto, a presença do parasita irá gerar abortos ou nascimentos de bezerros infectados. Já a forma endógena, ocorre devido à reativação de cistos que já estavam no animal antes da gestação, com a conversão dos bradizoítos presentes nos cistos em taquizoítos os quais são liberados na circulação ganhando a placenta e infectando o feto (Carvalho et al., 2014; Goodswen et al., 2013; Megid et al., 2016).

Vale salientar a relevância da transmissão transplacentária endógena na manutenção da doença no rebanho, visto que, aproximadamente $95 \%$ das vacas soropositivas irão abortar ou gerar bezerros infectados (Diniz et al., 2019; McAllister, 2016). Caracteriza-se pela transmissão do agente via materno fetal, em que a mãe pode infectar fetos por sucessivas gestações, culminando assim, na persistência do agente no rebanho (Maldonado Rivera et al., 2020; Margarido et al., 2008; Megid et al., 2016; $\underline{\text { Santos et }}$ al., 2011).

A transmissão horizontal é caracterizada quando os hospedeiros intermediários, quando assim infectados, adquirem os oocistos esporulados no ambiente, em fômites, alimentos contaminados, restos fetais e a partir da ingestão, desta forma, o protozoário se desenvolve em cistos teciduais formando bradizoítos ou fluídos com taquizoítos, no momento em que os oocistos atingem o estômago do hospedeiro intermediário, eles se rompem por ação mecânica liberando os esporozoítos no lúmen intestinal. Estes esporozoítos penetram nos tecidos e realizam multiplicação assexuada gerando taquizoítos. Os taquizoítos desenvolvem-se no interior de vacúolos parasitófaros, originando novos protozoários. Logo em seguida ocorre a ruptura da célula hospedeira e liberação dos taquizoítos

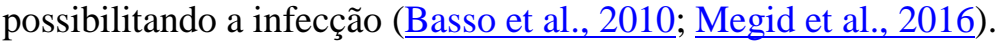

\section{Aspectos epidemiológicos}

Diversos estudos soroepidemiológicos já foram conduzidos no Brasil para determinar a soroprevalência do $N$. caninum em bovinos (Quadro 1). A presente revisão identificou uma soroprevalência média de $32,7 \%$, em 45 estudos levantados, com frequência mínima registrada de $2,45 \%$ (Rodrigues et al., 2016) e máxima de 100\% (Zanatto et al., 2019), durante um período de 15 anos de levantamento (2005-2020). Essa variação pode ocorrer devido aos fatores de risco inerentes a cada região, como microclima, presença de reservatórios silvestres, imunidade individual e do rebanho, presença de comorbidades, padrão racial, tipo de criação, idade dos animais, bem como, o manejo nutricional e sanitário.

A soro prevalência média em bovinos demonstra que possivelmente a doença está amplamente difundida no país, sendo considerada doença reprodutiva comum e provavelmente subestimada devido à elevada quantidade de animais assintomáticos, embora cronicamente afetados. Estima-se em escala 
global que abortos causados pelo $N$. caninum causem uma perda anual estimada maior que US\$1.298 bilhões, destes 75\% destinam-se a bovinocultura de leite (Reichel et al., 2013).

Quadro 1. Estudos sorológicos de Neospora caninum em bovinos no Brasil, de 2005 a 2020.

\begin{tabular}{|c|c|c|c|c|}
\hline Referência & $\mathrm{n}^{*}$ & Soro-prevalência, $\% * *$ & Estado & Técnica \\
\hline Ogawa et al. (2005) & 385 & $12(45 / 385)$ & Paraná & RIFI \\
\hline$\underline{\text { Sartor et al. (2005) }}$ & 913 & $27(246 / 913)$ & São Paulo & ELISA \\
\hline Corbellini (2005) & 1549 & $17,8(276 / 1549)$ & Rio Grande do Sul & RIFI \\
\hline$\underline{\text { Melo et al. (2006) }}$ & 930 & $30,4(283 / 930)$ & Goiás & RIFI \\
\hline Munhoz et al. (2006) & 563 & $23,2(131 / 563)$ & Rio de Janeiro & ELISA \\
\hline Oshiro et al. (2007) & 2448 & $14,9(365 / 2448)$ & Mato grosso do Sul & RIFI \\
\hline Oshiro et al. (2007) & 205 & $69,80(143 / 205)$ & Mato Grosso do Sul & RIFI \\
\hline$\underline{\text { Silva et al. (2008) }}$ & 469 & $31,7(163 / 469)$ & Pernambuco & RIFI \\
\hline Guedes et al. (2008) & 559 & $91,2(510 / 559)$ & Minas Gerais & RIFI \\
\hline Dittrich et al. (2008) & 1263 & $33(423 / 1263)$ & Paraná & ELISA \\
\hline Benetti et al. (2009) & 932 & $53,50(498 / 932)$ & Mato Grosso & RIFI \\
\hline$\underline{\text { Munhoz et al. (2009) }}$ & 563 & $23,3(131 / 563)$ & Rio de Janeiro & ELISA \\
\hline$\underline{\text { Santos et al. (2009) }}$ & 534 & $46,25(247 / 234)$ & Minas Gerais & RIFI \\
\hline Chiebao (2010) & 1039 & $17,1(178 / 1039)$ & Pará & RIFI \\
\hline Teixeira et al. (2010) & 812 & $50,7(412 / 812)$ & Maranhão & RIFI \\
\hline Andreotti et al. (2010) & 1098 & $62,50(686 / 1098)$ & Mato Grosso do Sul & RIFI \\
\hline Martins et al. (2011) & 192 & $25,0(48 / 192)$ & Tocantins & RIFI \\
\hline Marques et al. (2011) & 159 & $15,10(24 / 159)$ & Paraná & ELISA \\
\hline$\underline{\text { Sousa et al. (2012) }}$ & 1004 & $76,7(770)$ & Alagoas & RIFI \\
\hline Piagentini et al. (2012) & 615 & $32,0(197 / 615)$ & São Paulo & ELISA \\
\hline$\underline{\text { Martins et al. (2012) }}$ & 309 & $20,4(63 / 309)$ & Paraná & RIFI \\
\hline Moura et al. (2013) & 573 & $23,1(132 / 573)$ & Santa Catarina & RIFI \\
\hline Bruhn et al. (2013) & 1204 & $21,6(260 / 1204)$ & Minas Gerais & RIFI \\
\hline Nascimento et al. (2014) & 76 & $30,3(23 / 76)$ & Paraná & ELISA \\
\hline Vilas Boas (2014) & 621 & $10,61(66 / 621)$ & Rondônia & RIFI \\
\hline$\underline{\text { Ramos et al. (2016) }}$ & 316 & $19,6(62 / 316)$ & Pernambuco & RIFI \\
\hline$\underline{\text { Klauck et al. (2016) }}$ & 130 & $43,8(57 / 130)$ & Santa Catarina & RIFI \\
\hline Lorenzett et al. (2016) & 299 & $7,7(23 / 299)$ & Santa Catarina & RIFI \\
\hline$\underline{\text { Rodrigues et al. (2016) }}$ & 489 & $2,45(12 / 489)$ & Mato Grosso & RIFI \\
\hline$\underline{\text { Silva et al. (2017) }}$ & 500 & $52(260 / 500)$ & Pará & RIFI \\
\hline Fávero et al. (2017) & 1518 & $30,6(465 / 1518)$ & Santa Catarina & RIFI \\
\hline$\underline{\text { Curci (2017) }}$ & 945 & $35,13(332 / 945)$ & São Paulo & RIFI \\
\hline Macedo et al. (2017) & 41 & $51,2(21 / 41)$ & Santa Catarina & ELISA \\
\hline Nicolino et al. (2017) & 2915 & $21,9(638 / 2915)$ & Minas Gerais & ELISA \\
\hline$\underline{\text { Santin et al. (2017) }}$ & 120 & $76,6(92 / 120)$ & Goiás/Tocantins & ELISA \\
\hline Padilha et al. (2017) & 507 & $13,81(70 / 507)$ & Santa Catarina & RIFI \\
\hline$\underline{\text { Porto et al. (2018) }}$ & 84 & $15,47(13 / 84)$ & Paraná & RIFI \\
\hline$\underline{\text { Oliveira et al. (2018) }}$ & 255 & $27,06(69 / 255)$ & Piauí & RIFI \\
\hline Nasciutti et al. (2018) & 740 & $18,4(136 / 740)$ & Minas Gerais & ELISA \\
\hline$\underline{\text { Schmidt et al. (2018) }}$ & 2452 & $25,44(624 / 2452)$ & Mato Grosso & RIFI \\
\hline$\underline{\text { Snak et al. (2018) }}$ & 600 & $23,67(142 / 600)$ & Paraná & RIFI \\
\hline Appelt et al. (2019) & 262 & $32,82(86 / 262)$ & $\mathrm{RS} / \mathrm{SC}$ & \\
\hline Bastos et al. (2019) & 400 & $15(60 / 400)$ & São Paulo & RIFI \\
\hline Guerra et al. (2019) & 401 & $9,2(37 / 401)$ & Paraná & RIFI \\
\hline \multirow{4}{*}{ Zanatto et al. (2019) } & \multirow{4}{*}{102} & $85(17 / 20)$ & Goiás, & \multirow{4}{*}{ ELISA } \\
\hline & & $93(30 / 32)$ & São Paulo & \\
\hline & & $85(34 / 40)$ & Minas Gerais & \\
\hline & & $100(10 / 10)$ & Mato Grosso do Sul & \\
\hline
\end{tabular}

$\mathrm{n} *$ : número de bovinos avaliados no estudo; **Soro prevalência determinada pelo $\mathrm{n}$ de bovinos reagentes/n de bovinos avaliados; Técnica diagnóstica ELISA: Ensaio de Imunoadsorção Enzimático; RIFI: Reação de Imunofluorescência Indireta; RS: Rio Grande do Sul; SC: Santa Catarina 
Estimulam-se assim a realização de inquéritos epidemiológicos para determinarem o impacto da neosporose sob os índices reprodutivos do país, que visem identificar principalmente as vacas que são responsáveis por manter o patógeno nas propriedades rurais devido ser mantenedoras da transmissão endógena, com ativação da forma latente, via transplacentária por várias gestações. Estudos comprovam que aproximadamente 95\% das vacas soropositivas irão abortar ou gerar bezerros infectados (Diniz et al., 2019; McAllister, 2016).

A construção do cenário nacional da presente revisão corrobora com a meta-análise realizada por Ribeiro et al. (2019) que determinaram uma prevalência de 24\% na América do Sul, com significante correlação da infecção com abortos em rebanhos bovinos, assim como, Megid et al. (2016) destacaram que os estudos de soro prevalência de neosporose em bovinos infectados no Brasil esteja entre 20 a 30\%. Os principais fatores de risco apontados nessa meta-análise foram presença de cães nas fazendas, tipo de sistema de produção, identificando a bovinocultura leiteira mais susceptível frente à destinada ao corte, assim como detectado por Sartor et al. (2005). A ausência de teste diagnóstico na introdução de novos animais na em rebanhos leiteiros foi considerado um fato de risco relevante (Appelt et al., 2019).

Chiebao (2010) relatou que os fatores de risco em rebanhos bovinos associadas a $N$. caninum são a presença de cães, a criação de aves domésticas, o fornecimento de silagem e o tamanho da propriedade. Schmidt et al. (2018) em estudo conduzido no Pantanal, norte do Brasil, identificaram também a presença de cães e abortamento das vacas, assim como Nicolino et al. (2017) e Vilas Boas (2014), e associaram a soropositividade do rebanho avaliado também a idade $\leq 6$ anos e venda de bovinos para reprodução. Já Snak et al. (2018), além do histórico de aborto, apontaram em sua pesquisa outros fatores de risco, com raça e produção leiteira, apontaram que vacas da raça Jersey e baixa produção de leite como fatores significativos na ocorrência de animais sororeagentes.

Souza et al. (2019) em estudo realizado no Rio Grande do Sul, pelo método ELISA em três diferentes regiões encontraram uma soro prevalência de $21,8 \%$ a $35 \%$, sendo o aborto apontado como fator de risco para a doença, assim como Bruhn et al. (2013) que também identificaram repetição de cio e anestro em estudo realizado no estado de Minas Gerais.

\section{Manifestações clínicas}

A neosporose é caracterizada por apresentar um polimorfismo clínico, desde animais assintomáticos, em sua maioria, até pacientes com quadros neurológicos de ataxia, convulsão, paresia e paralisias (Megid et al., 2016). A principal manifestação clínica quando presente em bovinos é caracterizado pelo aborto, entre o quinto e o sexto mês de gestação, o que pode variar de acordo com o momento da parasitemia da vaca, podendo ocorrer em qualquer momento da gestação, além disso, os fetos podem morrer no útero e serem mumificados ou autolisados, apresenta sinais nervosos e queda na produção leiteira (Almeria et al., 2010; Llano, 2013; Maldonado Rivera et al., 2020; Reiterová et al., 2009). Dias et al.(2014) relatam que vacas de corte apresenta bezerros ao nascimento prematuros e com peso reduzido.

Quando os bezerros soropositivos (com até dois meses) apresentam manifestações clínicas, relacionam-se com disfunção neuro motora, podem apresentar ataxia, paralisia de membros com hipertensão rígida, reflexos patelares atenuados, convulsões e alteração do nível de consciência. Em recém-nascidos são observados exolftalmia ou assimetria dos olhos, hidrocefalia, estreitamento da medula espinhal, hipoplasia cerebelar e anormalidades do sistema nervoso central (lmeria et al., 2010).

\section{Diagnóstico}

Oshiro et al. (2007) destacam que o diagnóstico de neosporose bovina se fundamenta na verificação do histórico do rebanho (anamnese detalhada), manifestações clínicas, além de realização de exames complementares. Para se ter a confirmação diagnóstica é necessária a realização de testes laboratoriais (Megid et al., 2016).

O diagnóstico laboratorial da neosporose pode ser feito por métodos sorológicos como ELISA, RIFI, NAT, LAT e Immunoblotting, com intuito de identificar e eliminar os animais positivos do rebanho. Pode ser realizado ainda por técnicas moleculares utilizando os exames de imunohistoquímica, PCR, histopatológicas com uso de amostras de eleição na coleta, como placenta, líquidos fetais, cérebro, 
coração e fígado. E ainda pode-se realizar o isolamento do patógeno, podendo utilizar cultivo celular em camundongos (Carvalho et al., 2014; Margarido et al., 2008; Silva et al., 2013; Snak \& Osaki, 2019).

O exame de ELISA é o principal método indireto utilizado no diagnóstico sorológico, apresentando uma objetividade maior na interpretação dos resultados além de rapidez na realização, quando comparado a imunofluorescência indireta (Dubey et al., 2002). São inúmeros os estudos epidemiológicos de infecção por $N$. caninum realizados utilizando testes sorológicos (Quadro 1). Contudo, a identificação de animais soropositivos para $N$. caninum pode ser prejudicada devido à reatividade cruzada com outros protozoários Toxoplasmatíneos, principalmente $N$. hughesi e Hammondiaheydorni e a falta de padronização dos testes sorológicos, com diferentes pontos de corte e antígenos distintos, podendo conduzir a limitações diagnósticas (Cerqueira-Cézar et al., 2017).

$\mathrm{Na}$ lista de diagnóstico diferencial, Megid et al. (2016) e Snak \& Osaki (2019) elencam doenças reprodutivas como brucelose, diarreia viral bovina (DVB), rinotraqueíte infecciosa bovina (RIB), mielo encefalite protozoárica causada por Toxoplasma gondii e leptospirose, mediante ao polimorfismo clínico que a neosporose pode apresentar dificultando o estabelecimento do diagnóstico.

\section{Tratamentos}

Estudos realizados por Kritzner et al. (2002) em bezerros utilizando o ponazuril, obtiveram resultados positivos com efeito terapêutico sobre os taquizoítos do $N$. caninum. A infecção ocorreu de forma experimental, posteriormente foi realizando a aplicação de $20 \mathrm{mg} / \mathrm{kg}$ de ponazuril a $5 \%$ com a primeira dose sendo feita 24 horas após a infecção, a repetida a cada 24 horas por 6 dias, não apresentaram mais o parasita no cérebro ou em outros órgãos dos animais tratados com esse fármaco. Entretanto, o tratamento em bovinos parece ser inviável economicamente, devido à necessidade de utilização dos quimioterápicos de forma preventiva e por um longo período, elevando os riscos do aparecimento de resíduos no leite e na carne produzida (Dubey \& Schares, 2011), enaltecendo assim, a aplicação de método de controle e profilaxia da doença.

Outros autores relatam o uso de sulfadiazina, pirimetamina e clindamicina para o tratamento do $N$. caninum, no entanto, tem sua utilização bastante contestada devido ao custo e a eficácia dos protocolos, sendo necessário analisar criteriosamente qual a melhor diretriz a se tomar para realização do tratamento (Dubey et al., 2002).

A utilização de monensina com liberação lenta em vacas com o intuito de alterar o curso da infecção obteve resultados inconclusivos. Em estudo em vacas secas alimentadas com monensina pode-se observar redução do risco de infectividade protozoárica (Vanleeuwen et al., 2010).

\section{Profilaxia e controle}

Uma das formas mais eficazes de controle na introdução da infecção no rebanho são a realização de testes diagnósticos e atestado negativo para animais provindos de outras propriedades (Megid et al., 2016). São sugeridas também como alternativa de controle, algumas práticas de manejo relacionadas à interrupção do ciclo de transmissão que serão discutidas a seguir.

Em estudo executado por Silva et al. (2008) demonstraram que cerca de $46 \%$ da casuística de $N$. caninum ocorreu em propriedades que não adotavam medidas de biosseguridade, já os locais que mantiveram medidas aceitáveis pôde-se observar uma redução dessa prevalência para 21\%. Medidas de biossegurança como restringir acesso de cães a fetos abordados, tecidos infectados e fluidos de placentas, uso somente de vacas soronegativas na transferência de embrião, evitar acesso de outros animais a bebedouros e comedouros, uso de maternidades individuais, controle de roedores e a implementação de um programa de vacinação pode resultar de forma considerável na minimização dos prejuízos econômicos (Dias et al., 2014; Dubey et al., 2007; Reiterová et al., 2009).

Caso se tenha um resultado positivo é fundamental realizar uma avaliação completa do rebanho para verificar a quantidade de animais acometidos, além de determinar a principal forma de transmissão ocorrida na propriedade e destino dos animais infectados (Megid et al., 2016). Medidas de manejo sanitário deverão ser adotadas sendo imprescindíveis para o controle e prevenção da neosporose bovina (Megid et al., 2016). Aconselha-se também a utilização da técnica de transferência de embrião, devido 
ao fato de os embriões pré-implantados serem resistentes à infecção pelo $N$. caninum (Bielanski et al., 2002). Outra medida recomendada é o controle adequado da população canina da propriedade, para evitar o contato destes com os bovinos, manter os silos bem fechados, e os hospedeiros definitivos afastados, juntamente com a eliminação de maneira correta de material advindo de abortos (preferencialmente incinerados), uma vez que, são fatores que podem propiciar novas infecções (Lindsay et al., 1999; Sánchez et al., 2018).

Manejo das pastagens com rotação de pastos e controle da altura de entrada e saída do pasto adequada permitirá uma maior incidência solar e possível dessecação do oocisto no ambiente. O destino responsável das carcaças e restos placentários, os quais deverão se enterrados ou incinerados, impactam significantemente no controle da neosporose (Megid et al., 2016).

A neosporose não tem significância em saúde pública, pois trata-se de uma doença exclusiva de animais, não sendo considerada uma zoonose (Cerqueira-Cézar et al., 2017). No entanto, relatos de que $21,6 \%(81 / 310)$ dos pacientes portadores do vírus da imunodeficiência humana apresentavam anticorpos anti- $N$. caninum (RIFI, 1:50) em estudo realizado por Oshiro et al. (2015).

Diante das diferentes perspectivas, como a expansão do espectro de hospedeiros de $N$. caninum, ainda alguns não elucidados completamente seus papéis no ciclo biológico do agente, indução de reações cruzadas por protozoários afins, bem como, outras prováveis vias de transmissão do parasito e a carência de vacinas eficientes devido à escassez de estudos moleculares, torna-se ainda mais complexo o controle da neosporose. As medidas profiláticas sugeridas consistem na redução da exposição dos animais a possíveis fontes de infecção. Existe uma demanda crescente para que seja elaborada uma vacina eficiente a fim de prevenir abortamentos em bovinos e impedir a excreção de oocistos nos hospedeiros definitivos (Cerqueira-Cézar et al., 2017).

\section{Considerações finais}

A neosporose bovina está amplamente distribuída pelo Brasil, em alguns estudos de bovinocultura de exploração leiteira foram identificadas soroprevalências acima de 90\%, associadas ao histórico de aborto, presença de cães, tipo de criação, idade e ausência de teste diagnóstico na introdução de novos animais. A ocorrência média da neosporose encontrada nos estudos foi de 32,7\% (2,45\%-100\%). Esses achados justificam a implementação de estratégias de mitigação de riscos para reduzir a prevalência da neosporose bovina no Brasil, em busca de melhorias dos índices reprodutivos do rebanho nacional.

\section{Referências bibliográficas}

Almeria, S., Araujo, R., Tuo, W., Lopez-Gatius, F., Dubey, J. P., \& Gasbarre, L. C. (2010). Fetal death in cows experimentally infected with Neospora caninum at 110 days of gestation. Veterinary Parasitology, 169(3-4), 304-311. DOI: https://doi.org/10.1016/j.vetpar.2009.12.044

Almería, S., \& López-Gatius, F. (2013). Bovine neosporosis: clinical and practical aspects. Research in Veterinary Science, 95(2), 303-309. DOI: https://doi.org/10.1016/j.rvsc.2013.04.008

Andreotti, R. (2001). Neosporose: um possível problema reprodutivo para o rebanho bovino. Hora Veterinária, 122, 65-67.

Andreotti, R., Barros, J. C., Pereira, A. R., Oshiro, L. M., Cunha, R. C., \& Figueiredo Neto, L. F. (2010). Association between seropositivity for Neospora caninum and reproductive performance of beef heifers in the Pantanal of Mato Grosso do Sul, Brazil. Revista Brasileira de Parasitologia Veterinária, 19(2), 119-123. DOI: https://doi.org/10.1590/s1984-29612010000200010

ANUALPEC. (2019). Anuário da Pecuária Brasileira (20th ed., Vol. 1). Instituto FNP.

Appelt, M. A., Silva, A. S., Cazarotto, C. J., Machado, G., Rodrigues, R. S., Norbury, L. J., Baldissera, M. D., Alba, D. F., Gris, A., \& Mendes, R. E. (2019). Cholinesterase as an inflammatory marker of subclinical infection of dairy cows infected by Neospora caninum and risk factors for disease. Comparative Immunology, Microbiology and Infectious Diseases, 66, 101330. DOI: https://doi.org/10.1016/j.cimid.2019.101330

Basso, W., Schares, S., Minke, L., Bärwald, A., Maksimov, A., Peters, M., Schulze, C., Müller, M., Conraths, F. J., \& Schares, G. (2010). Microsatellite typing and avidity analysis suggest a common 
source of infection in herds with epidemic Neospora caninum-associated bovine abortion. Veterinary Parasitology, 173(1-2), 24-31. DOI: https://doi.org/10.1016/j.vetpar.2010.06.009

Bastos, S. B., Martins, T. A., Sammi, A. S., Martins, F. D. C., Cunha, I. A. L., Barros, L. D., Zulpo, D. L., \& Garcia, J. L. (2019). Neospora caninum: seroepidemiology of cows and dogs from milk farms of Cunha, São Paulo, Brazil. Semina: Ciências Agrárias, 40(6Supl2), 3123-3132. DOI: http://dx.doi.org/10.5433/1679-0359.2019v40n6Supl2p3123

Benetti, A. H., Schein, F. B., Santos, T. R., Gilson Hélio, T., Costa, A. J., Mineo, J. R., Lobato, J., Silva, D. A. de O., \& Gennari, S. M. (2009). Pesquisa de anticorpos anti-Neospora caninum em bovinos leiteiros, cães e trabalhadores rurais da região Sudoeste do Estado de Mato Grosso. Revista Brasileira de Parasitologia Veterinária, 18, 29-33. DOI: https://doi.org/10.4322/rbpv.018e1005

Bielanski, A., Robinson, J., \& Phipps-Todd, B. (2002). Effect of Neospora caninum on in vitro development of preimplantation stage bovine embryos and adherence to the zona pellucida. The Veterinary Record, 150(10), 316-318. DOI: https://doi.org/10.1136/vr.150.10.316

Bruhn, Fábio Rapahel Pascoti, Teófilo, T. S., Guimarães, A. K. V., Lima, R. F., Andrade, G. S., \& Guimarães, A. M. (2012). Neosporose em ruminantes. PUBVET, 6, Art-1270. DOI: https://doi.org/10.22256/pubvet.v16n2.1276

Bruhn, Fábio Raphael Pascoti, Daher, D. O., Lopes, E., Barbieri, J. M., Rocha, C. M. B. M., \& Guimarães, A. M. (2013). Factors associated with seroprevalence of Neospora caninum in dairy cattle in southeastern Brazil. Tropical Animal Health and Production, 45(5), 1093-1098. DOI: https://doi.org/10.1007/s11250-012-0330-y

Carvalho, R. P., Rabbers, A. S., Dutra, H. T., Silva, K. S., Batista, J. F., Lima, C. R. de O., \& Rabelo, R. E. (2014). Neosporose bovina-revisão de literatura. Revista Científica de Medicina Veterinária, 23(7), 1-23.

Cerqueira-Cézar, C. K., Calero-Bernal, R., Dubey, J. P., \& Gennari, S. M. (2017). Tudo sobre neosporose no Brasil. Revista Brasileira de Parasitologia Veterinária, 26(3), 253-279.

Chiebao, D. P. (2010). Frequência de anticorpos anti-Neospora caninum, anti-Brucella abortus e antiLesptospira spp. em bovinos do Estado do Pará: estudo de possíveis variáveis para ocorrência de infecção. Universidade de São Paulo.

Corbellini, L. G. (2005). Neosporose bovina: estudo de fatores de risco em 60 propriedades leiteras no estado do Rio Grande do Sul e levantamento de causas de aborto bovino com ênfase em Neospora caninum. Acta Scientiae Veterinariae, 33(2), 231-232. DOI: https://doi.org/10.22456/1679$\underline{9216.14837}$

Curci, V. C. M. (2017). Soroprevalência da neosporose em bovinos de leite criados em sistema de agricultura familiar. Revista Acadêmica Ciência Animal, 15(Suppl 2), 37-38. DOI: https://doi.org/10.7213/academica.15.s02.2017.18

Dias, L. R. O., Ferraz, H. T., Viu, M. A. de O., Lopes, D. T., \& Viu, A. F. M. (2014). Doenças parasitárias reprodutivas em bovinos-neosporose. PUBVET, 8(3), 1671. DOI: https://doi.org/10.22256/pubvet.v8n3.1671

Diniz, L. V. A., Minutti, A. F., Nino, B. S. L., Costa, L. R., Bosculo, M. R. M., de Almeida, B. F. M., Garcia, J. L., \& Barros, L. D. (2019). Vertical transmission of Neospora caninum in bovine fetuses from a slaughterhouse in Brazil. Tropical Animal Health and Production, 51(6), 1751-1755. DOI: https://doi.org/10.1007/s11250-019-01828-y

Dittrich, R. L., Machado Júniorr, P. C., Fridlund, N., Richartz, R. R., Ferreira., F. M., Patrício, L. F., \& Pieppe, M. (2008). Determinação e correlação de anticorpos anti-Neospora caninum em bovinos e cães do Paraná, Brasil. Revista Brasileira de Parasitologia Veterinária, 17(1), 191-195.

Donahoe, S. L., Lindsay, S. A., Krockenberger, M., Phalen, D., \& Šlapeta, J. (2015). A review of neosporosis and pathologic findings of Neospora caninum infection in wildlife. International Journal for Parasitology: Parasites and Wildlife, 4(2), 216-238. DOI: https://doi.org/10.1016/j.ijppaw.2015.04.002

Dubey, J. P., Barr, B. C., Barta, J. R., Bjerkås, I., Björkman, C., Blagburn, B. L., Bowman, D. D., Buxton, D., Ellis, J. T., \& Gottstein, B. (2002). Redescription of Neospora caninum and its 
differentiation from related coccidia. International Journal for Parasitology, 32(8), 929-946. DOI: https://doi.org/10.1016/s0020-7519(02)00094-2

Dubey, J. P., Carpenter, J. L., Speer, C. A., Topper, M. J., \& Uggla, A. (1988). Newly recognized fatal protozoan disease of dogs. Journal of the American Veterinary Medical Association, 192(9), 12691285.

Dubey, J. P., \& Schares, G. (2011). Neosporosis in animals - the last five years. Veterinary Parasitology, 180(1-2), 90-108. DOI: https://doi.org/10.1016/j.vetpar.2011.05.031

Dubey, J. P., Schares, G., \& Ortega-Mora, L. M. (2007). Epidemiology and control of neosporosis and Neospora caninum. Clinical Microbiology Reviews, 20(2), 323-367.

Fávero, J. F., Silva, A. S., Campigotto, G., Machado, G., Barros, L. D., Garcia, J. L., Vogel, F. F., Mendes, R. E., \& Stefani, L. M. (2017). Risk factors for Neospora caninum infection in dairy cattle and their possible cause-effect relation for disease. Microbial Pathogenesis, 110, 202-207. DOI: 10.1016/j.micpath.2017.06.042

Flores, E. F. (2012). Virologia veterinária. Universidade Federal de Santa Maria.

Goodswen, S. J., Kennedy, P. J., \& Ellis, J. T. (2013). A review of the infection, genetics, and evolution of Neospora caninum: from the past to the present. Infection, Genetics and Evolution, 13, 133-150. DOI: $\underline{10.1016 / j . m e e g i d .2012 .08 .012}$

Guedes, M. H. P., Guimarães, A. M., Rocha, C. M. B. M., \& Hirsch, C. (2008). Freqüência de anticorpos anti-Neospora caninum em vacas e fetos provenientes de municípios do sul de Minas Gerais. Revista Brasileira de Parasitologia Veterinária, 17(4), 189-194. DOI: https://doi.org/10.1590/s198429612008000400004

Guerra, J. L., Okano, W., Bogado, A. L. G., Nino, B. de S. L., Martins, F. D. C., Cardim, S. T., Barros, L. D., \& Garcia, J. L. (2019). Anti-Neospora caninum antibodies in beef cattle from the northern region of Paraná state, Brazil. Ciência Rural, 49(5), 1-4. DOI: https://doi.org/10.1590/0103$8478 \mathrm{cr} 20180869$

Klauck, V., Machado, G., Pazinato, R., Radavelli, W. M., Santos, D. S., Berwaguer, J. C., Braunig, P., Vogel, F. F., \& Silva, A. S. (2016). Relation between Neospora caninum and abortion in dairy cows: Risk factors and pathogenesis of disease. Microbial Pathogenesis, 92, 46-49. DOI: https://doi.org/10.1016/j.micpath.2015.12.015

Kritzner, S., Sager, H., Blum, J., Krebber, R., Greif, G., \& Gottstein, B. (2002). An explorative study to assess the efficacy of toltrazuril-sulfone (ponazuril) in calves experimentally infected with Neospora caninum. Annals of Clinical Microbiology and Antimicrobials, 1(1), 4.

Lindsay, D. S., Dubey, J. P., \& McAllister, M. (1999). Neospora caninum and the potential for parasite transmission. Compendium on Continuing Education for the Practicing Veterinarian, 21(4), 317321.

Llano, H. A. B. (2013). Revisão e situação atual da brucelose e leptospirose em bovinos no Brasil e na Colômbia. Seminário Apresentado Junto à Disciplina Seminários Aplicados Do Programa de PósGraduação Em Ciência Animal Da Escola de Veterinária e Zootecnia Da Universidade Federal de Goiás. Goiânia.

Lorenzett, M. P., Lucca, N. J., Henker, L. C., Machado, G., Gomes, D. C., Mendes, R. E., Driemeier, D., \& Casagrande, R. A. (2016). Ocorrência de anticorpos anti-Neospora caninum em bovinos leiteiros no oeste do estado de Santa Catarina, Brasil. Brazilian Journal of Veterinary Medicine, 38(3), 243-249.

Macedo, C. A. B., Macedo, M. F. S. B., Miura, A. C., Taroda, A., Cardim, S. T., Innes, E. A., Katzer, F., Cantón, G. J., Chianini, F., \& Headley, S. A. (2017). Ocorrência de abortamentos induzidos por Neospora caninum em vacas de leite de Santa Catarina, sul do Brasil. Revista Brasileira de Parasitologia Veterinária, 26(3), 292-298. DOI: https://doi.org/10.1590/s1984-29612017051

Maldonado Rivera, J. E., Vallecillo, A. J., Pérez, C. L., Cirone, K. M., Dorsch, M. A., Scioli, V., Hecker, Y., Fiorani, F., Canton, G. J., \& Moore, P. D. (2020). Bovine neosporosis in dairy cattle from the southern highlands of Ecuador. Regional Studies and Reports, 20(1), 1-15. DOI: https://doi.org/10.1016/j.vprsr.2020.100377 
Margarido, R. S., Lima, D. N., Monteiro, J. R., \& Neves, M. F. (2008). Neosporose. Revista Cientifica. Eletrônica de Medicina Veterinária, 6(11), 1-7.

Marques, F. A. C., Headley, A. S., Figueredo-Pereira, V., Taroda, A., Barros, L. D., Cunha, I. A. L., Munhoz, K., Bugni, F. M., Zulpo, D. L., \& Igarashi, M. (2011). Neospora caninum: evaluation of vertical transmission in slaughtered beef cows (Bos indicus). Parasitology Research, 108(4), 10151019. DOI: https://doi.org/10.1007/s00436-010-2146-X

Martins, A. A., Oliveira Zamprogna, T., Lucas, T. M., Cunha, I. A. L., Garcia, J. L., \& Silva, A. V. (2012). Frequency and risk factors for infection by Neospora caninum in dairy farms of Umuarama, PR, Brazil. Arquivos de Ciências Veterinárias e Zoologia Da UNIPAR, 15(2), 137-142.

Martins, N. É. X., Freschi, C. R., Baptista, F., Machado, R. Z., Freitas, F. L. C., \& Almeida, K. S. (2011). Ocorrência de anticorpos anti-Neospora caninum em vacas lactantes do município de Araguaína, estado do Tocantins, Brasil. Revista de Patologia Tropical/Journal of Tropical Pathology, 40(3), 231-238. DOI: https://doi.org/10.5216/rpt.v40i3.15973

McAllister, M. M. (2016). Diagnosis and control of bovine neosporosis. Veterinary Clinics: Food Animal Practice, 32(2), 443-463. DOI: https://doi.org/10.1016/j.cvfa.2016.01.012

Megid, J., Ribeiro, M. G., \& Paes, A. C. (2016). Doenças infecciosas em animais de produção e de companhia. Roca.

Melo, D. P. G., Silva, A. C., Ortega 'Mora, L. M., Bastos, S. A., \& Boaventura, C. M. (2006). Prevalência de anticorpos anti-Neospora caninum em bovinos das microrregiões de Goiânia e Anápolis, Goiás, Brasil. Revista Brasileira de Parasitologia Veterinária, 15(3), 105-109.

Moura, A. B., Silva, M. O., Farias, J. A., Vieira Neto, A., Souza, A. P., Sartor, A. A., Fonteque, J. H., \& Bunn, S. (2013). Neospora spp. antibodies in horses from two geographical regions of the state of Santa Catarina, Brazil. Revista Brasileira de Parasitologia Veterinária, 22(4), 597-601. DOI: https://doi.org/10.1590/s1984-29612013000400023

Munhoz, A. D., Flausino, W., Silva, R. T., Almeida, C. R., \& Lopes, C. W. (2006). Distribuição de anticorpos contra Neospora caninum em vacas leiteiras dos municípios de Resende e Rio Claro, Estado do Rio de Janeiro, Brasil. Revista Brasileira de Parasitologia Veterinária, 15(3), 101-104.

Munhoz, Alexandre D, Pereira, M. J. S., Flausino, W., \& Lopes, C. W. G. (2009). Neospora caninum seropositivity in cattle breeds in the South Fluminense Paraíba Valley, state of Rio de Janeiro. Pesquisa Veterinária Brasileira, 29(1), 29-32. DOI: https://doi.org/10.1590/s0100736x2009000100004

Nascimento, E. E., Sammi, A. S., Dos Santos, J. R., Nino, B. de S. L., Bogado, A. L. G., Taroda, A., Vidotto, O., \& Garcia, J. L. (2014). Anti-Neospora caninum antibody detection and vertical transmission rate in pregnant zebu beef cows (Bos indicus): Neospora caninum in pregnant beef cows (Bos indicus). Comparative Immunology, Microbiology and Infectious Diseases, 37(4), 267-270. DOI: https://doi.org/10.1016/j.cimid.2014.08.002

Nasciutti, N. R., Oliveira, P. M., Barbosa, V. M., Headley, S. A., Garcia, F. G., Silva, M. V, Ferreira, F. B., Mineo, T. W. P., \& Saut, J. P. E. (2018). Soroprevalência e fatores de risco associados ao herpesvírus bovino tipo 1, vírus da diarréia viral bovina em vacas leiteiras mestiças não vacinadas e Neospora caninum no município de Uberlândia. Semina: Ciencias Agrarias, 39(4), 1585-1594.

Nicolino, R. R., Oliveira, C. S. F., Lopes, L. B., Rodrigues, R. O., \& Haddad, J. P. A. (2017). Prevalence and risk factors associated with anti-Neospora caninum antibodies in dairy herds in the central region of Minas Gerais State, Brazil. Veterinary Parasitology: Regional Studies and Reports, 10, 71-74. DOI: https://doi.org/10.1016/j.vprsr.2017.08.008

Ogawa, L., Freire, R. L., Vidotto, O., Gondim, L. F. P., \& Navarro, I. T. (2005). Occurrence of antibodies to Neospora caninum and Toxoplasma gondii in dairy cattle from the northern region of the Paraná State, Brazil. Arquivo Brasileiro de Medicina Veterinária e Zootecnia, 57(3), 312-316. DOI: https://doi.org/10.1590/s0102-09352005000300006

Oliveira, R. P., Soares, N. M., Silva, J. G., Ribeiro-Andrade, M., Lira, N. S. C., Farias, M. P. O., \& Mota, R. A. (2018). Occurrence of anti-Neospora caninum antibodies in cattle in the dairy farming region of the state of Piauí, Brazil. Revista Brasileira de Parasitologia Veterinária, 27(4), 589-592. DOI: https://doi.org/10.1590/s1984-296120180046 
Oshiro, L. M., Matos, M. de F. C., Oliveira, J. M., Monteiro, L. A. R. C., \& Andreotti, R. (2007). Prevalence of anti-Neospora caninum antibodies in cattle from the state of Mato Grosso do Sul, Brazil. Revista Brasileira de Parasitologia Veterinária, 16(3), 133-138. DOI: https://doi.org/10.1590/s1984-29612007000300004

Oshiro, L. M., Castro, A. R. C. M., Freitas, S. Z., Cunha, R. C., Dittrich, R. L., Meirelles, A. C. F., \& Andreotti, R. (2015). Neospora caninum and Toxoplasma gondii serodiagnosis in human immunodeficiency virus carriers. Revista Da Sociedade Brasileira de Medicina Tropical, 48(5), 568-572. DOI: https://doi.org/10.1590/0037-8682-0151-2015

Padilha, M. A. C., Wasen, G., Souza, A. P., Milczewski, V., Luz, T. V. B., Sartor, A. A., Farias, J. A., \& Moura, A. B. (2017). Neospora caninum: Seroprevalence in beef cattle in the mountainous region of Santa Catarina, Brazil. Semina: Ciências Agrárias, 38(1), 273-281. DOI: https://doi.org/10.5433/1679-0359.2017v38n1p273

Piagentini, M., Moya-Araujo, C. F., Prestes, N. C., \& Sartor, I. F. (2012). Neospora caninum infection dynamics in dairy cattle. Parasitology Research, 111(2), 717-721. DOI: https://doi.org/10.1007/s00436-012-2891-0

Porto, Y. F., Pinto Neto, A., Bernardi, F., Possa, M. G., Mota, M. F., Martinez, A. C., Merlini, L. S., \& Berber, R. C. (2018). Occurrence of brucellosis, leptospirosis and neosporosis in cows with retained placenta in Southwest Paraná, Brazil. Pesquisa Veterinária Brasileira, 38(8), 1537-1542. DOI: https://doi.org/10.1590/1678-5150-pvb-5415

Ramos, I. A. S., Silva, R. J., Maciel, Thiago ArcoverdeSilva, J. A. B., Fidelis Junior, O. L. A., Soares, P. C., Machado, R. Z., André, M. R., \& Mendonça, C. L. (2016). Assessment of transplacental transmission of Neospora caninum in dairy cattle in the Agreste region of Pernambuco. Revista Brasileira de Parasitologia Veterinária, 25(4), 516-522. DOI: https://doi.org/10.1590/s198429612016055

Reichel, M. P., Alejandra Ayanegui-Alcérreca, M., Gondim, L. F. P., \& Ellis, J. T. (2013). What is the global economic impact of Neospora caninum in cattle - The billion dollar question. International Journal for Parasitology, 43(2), 133-142. DOI: https://doi.org/10.1016/j.ijpara.2012.10.022

Reiterová, K., Špilovská, S., Antolová, D., \& Dubinský, P. (2009). Neospora caninum, potential cause of abortions in dairy cows: the current serological follow-up in Slovakia. Veterinary Parasitology, 159(1), 1-6. DOI: https://doi.org/10.1016/j.vetpar.2008.10.008

Ribeiro, C. M., Soares, I. R., Mendes, R. G., Bastos, P. A. S., Katagiri, S., Zavilenski, R. B., Abreu, H. F. P., \& Afreixo, V. (2019). Meta-analysis of the prevalence and risk factors associated with bovine neosporosis. Tropical Animal Health and Production, 51(7), 1-18. DOI: https://doi.org/10.1007/s11250-019-01929-8

Rodrigues, R. S., Igarashi, M., Muraro, L. S., Gomes, A. H. B., Aguiar, D. M., dos Anjos Pacheco, T., Okano, W., Barros, M. P., \& Santos, M. D. (2016). The occurrence of anti-Neospora caninum in bovine female animals and bovine fetuses in Nossa Senhora do Livramento County, Mato Grosso. Semina: Ciências Agrárias, 37(6), 4161-4166. DOI: https://doi.org/10.5433/16790359.2016v37n6p4161

Sánchez, R., Ferre, I., Re, M., Vázquez, P., Ferrer, L. M., Murcia, J. B., Lunar, P. G. (2018). Segurança e eficácia do inibidor da quinase colidida BKI-1553 em ovelhas prenhes infectadas experimentalmente com taquizozitos de Neosporacaninum. International Journal for Parasitology: DrugsandDrugResistance, 8(1), 112-124.

Santin, A. P. I., Juliano, R. S., Silva, A. C., Oliveira, V. S. F., Junqueira-Kipnis, A. P., Brito, W. M. E. D. de, \& Fioravanti, M. C. S. (2017). Soroepidemiologia de Neospora caninum e Toxoplasma gondii em bovinos da raça curraleiro. Ciência Animal Brasileira, 18, 1-8. DOI: https://doi.org/10.1590/1089-6891v18e-44649

Santos, D. S., Andrade, M. P., Varaschin, M. S., Guimarães, A. M., \& Hirsch, C. (2011). Neospora caninum in bovine fetuses of Minas Gerais, Brazil: genetic characteristics of rDNA. Revista Brasileira de Parasitologia Veterinária, 20(4), 281-288. DOI: https://doi.org/10.1590/s198429612011000400005

Santos, R. R. D., Guimares, A. M., Rocha, C. M. B. M., \& Hirsch, C. (2009). Frequência de anticorpos 
anti-neospora caninum em bezerras e novilhas de rebanhos leiteiros na microrregião de lavras, MG. Ciência Animal Brasileira, 10(1), 271-280.

Sartor, I. F., Garcia Filho, A., Vianna, L. C., Pituco, E. M., Dal Pai, V., \& Sartor, R. (2005). Ocorrência de anticorpos anti-Neospora caninum em bovinos leiteiros e de corte da região de Presidente Prudente, SP. Arquivos Do Instituto Biológico, 72(4), 413-418.

Schmidt, A. C., Pacheco, T. A., Barddal, J. E. I., Oliveira, A. C. S., Aguiar, D. M., Negreiros, R. L., \& Pacheco, R. de C. (2018). Seroprevalence, spatial analysis and risk factors of infection with Neospora caninum in cattle in Brazil's northern Pantanal wetland. Revista Brasileira de Parasitologia Veterinária, 27(4), 455-463. DOI: https://doi.org/10.1590/s1984-296120180058

Silva, J. B., Nicolino, R. R., Fagundes, G. M., Bomjardim, H. A., Reis, A. S. B., Lima, D. H. S., Oliveira, C. M. C., Barbosa, J. D., \& Fonseca, A. H. (2017). Serological survey of Neospora caninum and Toxoplasma gondii in cattle (Bos indicus) and water buffaloes (Bubalus bubalis) in ten provinces of Brazil. Comparative Immunology, Microbiology and Infectious Diseases, 52, 30-35. DOI: https://doi.org/10.1016/j.cimid.2017.05.005

Silva, M. I. S., Almeida, M. Â. O., Mota, R. A., Pinheiro Júnior, J. W. P., \& Rabelo, S. S. A. (2008). Fatores de riscos associados à infecção por Neospora caninum em matrizes bovinas leiteiras em Pernambuco. Ciência Animal Brasileira, 9(2), 455-461.

Silva, S. P., Mota, R. A., Faria, E. B., Casseb, A. R., Casseb, L., \& Dias, H. L. T. (2013). Comparação das técnicas de ELISA indireto e Imunofluorescência indireta na detecção de anticorpos antiNeospora caninum em búfalas (Bubalus bubalis). Pesquisa Veterinária Brasileira, 33(4), 431-434. DOI: https://doi.org/10.1590/s0100-736x2013000400003

Snak, A., Garcia, F. G., Lara, A. A., Pena, H. F. J., \& Osaki, S. C. (2018). Neospora caninum in properties in the west region of Paraná, Brazil: prevalence and risk factors. Revista Brasileira de Parasitologia Veterinária, 27(1), 52-60. DOI: https://doi.org/10.1590/s1984-29612018001

Snak, A., \& Osaki, S. C. (2019). Uma revisão sobre três importantes agentes causadores de aborto em bovinos: Neospora caninum, Leptospira sp. e Trypanosoma vivax. Revista de Ciência Veterinária e Saúde Pública, 6(1), 160-195. DOI: https://doi.org/10.4025/revcivet.v6i1.39623

Sousa, M. E., Wagnner, J. N., Albuquerque, P. P. F., Souza Neto, O. L., Faria, E. B., Pinheiro Júnior, J. W., \& Mota, R. A. (2012). Soroprevalência e fatores de risco associados à infecção por Neospora caninum em bovinos leiteiros no estado de Alagoas. Pesquisa Veterinária Brasileira, 32(10), 1009_ 1013.

Souza, G. N., Pegoraro, L. M. C., Weissheimer, C. F., Fischer, G., Dellagostin, O. A., Bialves, T. S., Gindri, P. C., Lucas, R. M., Muller, L., \& Cavalcanti, F. (2019). Epidemiological situation and risk Factors to Infectious Diseases in Dairy Cattle Located in Different Mesoregions of the State of Rio Grande do Sul, Brazil, 2016/2017. Embrapa Clima Temperado-Artigo Em Periódico Indexado (ALICE).

Teixeira, W. C., Uzêda, R. S., Gondim, L. F. P., Silva, M. I. S., Pereira, H. M., Alves, L. C., \& Faustino, M. A. G. (2010). Prevalência de anticorpos anti-Neospora caninum (Apicomplexa: Sarcocystidae) em bovinos leiteiros de propriedades rurais em três microrregiões no estado do Maranhão. Pesquisa Veterinária Brasileira, 30(9), 729-734. DOI: https://doi.org/10.1590/s0100-736x2010000900004

Vanleeuwen, J. A., Haddad, J. P., Dohoo, I. R., Keefe, G. P., Tiwari, A., \& Scott, H. M. (2010). Risk factors associated with Neospora caninum seropositivity in randomly sampled Canadian dairy cows and herds. Preventive Veterinary Medicine, 93(2-3), 129-138. DOI: https://doi.org/10.1016/j.prevetmed.2009.11.013

Varandas, N. P., Rached, P. A., Costa, G. H. N., Souza, L. M., Castagnolli, K. C., \& Costa, A. J. (2001). Frequência de anticorpos anti-Neospora caninum e anti-Toxoplasma gondii em cães da região nordeste do Estado de São Paulo: correlação com neuropatias. Semina: Ciências Agrárias, 22(1), 105-111. DOI: https://doi.org/10.5433/1679-0359.2001v22n1p105

Vilas Boas, R. (2014). Prevalência e fatores de risco de anticorpos anti-Neosporacaninum em fêmeas bovinas leiteiras da agricultura familiar no município de Ji-Paraná, Rondônia. Dissertação (Mestrado em Ciências Animal) Universidade Federal do Mato Grosso. 
Zanatto, D. C. S., Gatto, I. R. H., Labruna, M. B., Jusi, M. M. G., Samara, S. I., Machado, R. Z., \& André, M. R. (2019). Coxiella burnetii associated with BVDV (Bovine Viral Diarrhea Virus), BoHV (Bovine Herpesvirus), Leptospira spp., Neospora caninum, Toxoplasma gondii and Trypanosoma vivax in reproductive disorders in cattle. Revista Brasileira de Parasitologia Veterinária, 28(2), 245257. DOI: https://doi.org/10.1590/s1984-29612019032

Recebido: 5 de abril, 2020.

Aprovado: 22 de maio, 2020

Disponível online: 28 de agosto, 2020.

Licenciamento: Este artigo é publicado na modalidade Acesso Aberto sob a licença Creative Commons Atribuição 4.0 (CC-BY 4.0), a qual permite uso irrestrito, distribuição, reprodução em qualquer meio, desde que o autor e a fonte sejam devidamente creditados. 